\title{
Rapid and reliable 25-OH vitamin D2 and 25-OH vitamin D3 measurements by multitasker LC-MS/MS
}

\author{
Ahmet C Gören $\odot^{* 1}$, Gözde Şahin $\odot^{2}$, İsmail Gümilcineli $\odot^{2}$ and Burcu Binici $\odot$ \\ ${ }^{1} T U ̈ B I T T A K ~ U M E$ (National Metrology Institute), Organic Chemistry Laboratories, 41470, \\ Gebze-Kocaeli, Türkiye \\ ${ }^{2}$ Zivak Technologies, Gürsel Mh. Eski Beşiktaş Cad. No: 44/1-2 34400, Kağıthane, İstanbul,
}

Türkiye

(Received February 16, 2018; Revised April 24, 2018; Accepted April 30, 2018)

\begin{abstract}
The 25-OH Vitamin D2 and 25-OH Vitamin D3 measurement method was validated using a Zivak Multitasker Fully Automated Sample Preparation and Injection System coupled to a MS detector. The current method incorporates all sample preparations, SPE clean up procedures, LC and MS facilities into an all in one system. This system can report patient data within 6 minutes including the SPE and Sample preparation and mass spectrometry analysis time. The correlation coefficient of the developed method was greater than 0.99 for measurands, 25-OHvitamin Ds, in the calibration range. The measurement uncertainty of the measurements were evaluated and found as $7.7 \%$ and $7.6 \%$ for $25-\mathrm{OH}$ vitamin D2 and 25-OH-vitamin D3, respectively.
\end{abstract}

Keywords: 25-OH Vitamin D2; 25-OH Vitamin D3; MS/MS; ZIVAK multitasker; method validation; uncertainty. (C) 2018 ACG Publications. All rights reserved.

\section{Introduction}

Vitamin D, also known as vitamin sunshine, is a fat soluble vitamin found in the forms of Vitamin D2 and Vitamin D3. Vitamin D2 is usually found in several vegetable based foods while Vitamin D3 is predominantly found in meat. Vitamin D3 is formed naturally by the exposure of our skin to UV radiation found in sunlight. The main function of Vitamin D in human metabolism is defined as regulating calcium and phosphorus levels in the bloodstream. There are an increasing amount of studies and views on the different metabolic functions of Vitamin D. Studies show that its` widespread impact and the need for its reliable and quick measurement is of paramount importance [1-6].

Vitamin D is metabolized in the human body to 25-hydroxyvitamin D3 [25-(OH)D3], 3-epi-25hydroxyvitamin D3 [3-epi-25(OH)D3] and 25-hydroxyvitamin D2 [25-(OH)D2] and these metabolites are commonly studied in human serums. The total concentration of $25(\mathrm{OH}) \mathrm{D} 2$ and $25(\mathrm{OH}) \mathrm{D} 3$ in human serums are generally within $16 \mathrm{ng} / \mathrm{g}-30 \mathrm{ng} / \mathrm{g}(40 \mathrm{nmol} / \mathrm{L}-75 \mathrm{nmol} / \mathrm{L})$ of which $90 \%$ is in the $25(\mathrm{OH}) \mathrm{D} 3 \mathrm{form}$ and

\footnotetext{
${ }^{*}$ Corresponding author: E-Mail: ahmetcgoren@yahoo.com
} 
Rapid and reliable 25-OH vitaminD's measurements

the remaining $10 \%$ is a combination of all other forms. Due to 3 -epi-25(OH)D3 being found only in trace amounts, it is not commonly studied in routine analyses carried out by clinical biochemists. Values below $12 \mathrm{ng} / \mathrm{mL}(30 \mathrm{nmol} / \mathrm{mL})$ of $25-\mathrm{OH}$ vitamin D are defined as a Vitamin D deficiency and point towards rickets in newly born babies and osteomalacia in adults [4-9]. A value of $12-20 \mathrm{ng} / \mathrm{mL}(20-50 \mathrm{nmol} / \mathrm{mL})$ is considered inadequate for bone and overall health in healthy individuals. A value of $20-50 \mathrm{ng} / \mathrm{mL}(50-125$ $\mathrm{nmol} / \mathrm{mL}$ ) is deemed adequate for a healthy adult however a value above this is evaluated to be serious and probable evidence for potential side effects.

The metrological examinations of vitamins and metabolites have been well established by metrology institutes and are used in clinical chemistry. The NIST provided service of SRM 972 to clinical laboratories in 2009 [10] and SRM 972a in 2013 [11] while UME CRM 1308 was provided by TUBITAK UME in 2016 [12]. While the various metrology institutes over the world report Vitamin D measurements with low uncertainties in the CCQM level, $2.3 \%$ to $6.8 \%$ for Vitamin D2 and $2.1 \%$ to $12.6 \%$ for Vitamin D3, the uncertainty values are generally not only not reported by second tier laboratories but also often report CV values between $10-30 \%$ [1-9].

It is seen from reported Vitamin D measurements using the common immunoassay (CLIA, EIA and RIA) method with HPLC methods have a higher range in measurement uncertainties in comparison to LCMS/MS measurements which have a lower range [7,8]. Therefore the ID-LC-MS/MS is accepted as the primary method of measuring Vitamin D and because of its accurate and precise measurements it is becoming increasingly popular in clinical laboratories [4-7].

The major obstacle in clinical laboratories is the preparation of samples which is time consuming, prone to error and an increase in uncertainty arising from reproducibility which in turn reduces reliability in the measurements. ZIVAK Vitamin D was developed to obtain quick and reliable measurements in the laboratory and 25(OH)D2 and 25(OH)D3 measurements were performed with the ZIVAK Vitamin D analyzer in order to overcome these obstacles. The validation of the instrument and kit was carried out and are reported in this study.

\section{Experimental}

\subsection{Standards and Chemicals}

UME CRM $1308(51.00 \pm 2.92(\mathrm{ng} / \mathrm{mL})$ for 25-OH-vitamin D2 and 49.76 $\pm 2.65(\mathrm{ng} / \mathrm{mL})$ for 25 OH-vitamin D3) obtained from TUBITAK UME. 25-OH-vitamin D3 lyophilized serums control produced by ZIVAK coded as ZV-3046-02S1-10, ZV-3046-02S2-10, ZV-3046-02S3-10 (Table 1). The Reagent 1 (ZV-304602R1-10) and Reagent 2 (ZV-304602R2-10) were obtained from ZIVAK Technologies together with the Mobile Phase A (ZV-3046-02-MP-10), SPE Buffer (ZV3046-02-MT-10) and washing solution (ZV3046-02-WB-10). Working lyophilized serum calibrators containing 25-OH Vitamin D2 and D3 were prepared in distilled water (v/v) based on the linearity range and all sample preparation steps, dilution, precipitation, vortexing, centrifuge and injection steps were done by using the ZIVAK Vitamin D Multitasker system with the software of AUTOMASS. The chemicals and reagents were stored in a refrigerator at $-20^{\circ} \mathrm{C}$ in glass containers.

Table 1. Lyophilized serum control materials for 25-OH-vitamin Ds

\begin{tabular}{llccc}
\hline The Code & Control Standard & Lot number & $\begin{array}{c}\text { 25-OH-VitD2 } \\
\text { (ng/mL) }\end{array}$ & $\begin{array}{c}\text { 25-OH-VitD3 } \\
\text { (ng/mL) }\end{array}$ \\
\hline ZV-3046-02S1-10 & Calibrator Level 1 & VD1702 S1 & 9.99 & 14.71 \\
ZV-3046-02S2-10 & Calibrator Level 2 & VD1702 S2 & 28.68 & 25.88 \\
ZV-3046-02S3-10 & Calibrator Level 3 & VD1702 S3 & 41.62 & 32.61 \\
ZV-3046-02S4-10 & Calibrator Level 4 & VD1702 S4 & 53.74 & 39.80 \\
\hline
\end{tabular}

\subsection{MS Parameters}

Waters Quattro Premier Tandem Quadrupole MS equipped with a heated electrospray ionization source (ESI) was used in positive mode as mass detector in the applied method. The capillary voltage was $3.5 \mathrm{kV}$. 
The details of the applied method were given in Table 2. The Zivak Multitasker Fully Automated Sample Preparation and Injection System were used with the Mobile Phase of the ZIVAK Vitamin D analysis kit.

Table 2. Mass Spectrometry parameters of applied MS/MS method

\begin{tabular}{lccccc}
\hline & $\begin{array}{c}\text { Parent } \\
(\boldsymbol{m} / \boldsymbol{z})\end{array}$ & $\begin{array}{c}\text { Daughter } \\
(\boldsymbol{m} / \boldsymbol{z})\end{array}$ & $\begin{array}{c}\text { Dwell } \\
\text { time (s) }\end{array}$ & Cone (V) & Collision (V) \\
25-OH-Vitamin D2 & 413.1 & 81.6 & 0.050 & 24 & 22 \\
25-OH-Vitamin D3 & 401.15 & 383.2 & 0.050 & 22 & 10 \\
25-OH-Vitamin D2-d6 (IS) & 407.15 & 158.75 & 0.050 & 22 & 28 \\
\hline
\end{tabular}

\subsection{Sample Preparation}

25-Hydroxyvitamin D2-D3 analysis was performed using the Zivak Multitasker Fully Automated Sample Preparation and Injection System connected to an MS system which is a Waters Quattro Premier Tandem Quadrupole MS equipped with a heated electrospray ionization source (ESI). The system was controlled with AUTOMASS software. The SPE purification was done automatically using ZV-3046CSPE-10, Zivak 25-Hydroxyvitamin D2-D3 Serum LC-MS/MS Trap Column Cartridge via AUTOMASS software and a Zivak 25-Hydroxyvitamin D2-D3 reversed phase column from Zivak Technologies, Turkey with a $50 \times 2 \mathrm{~mm} \times 3 \mu \mathrm{m}$ particle size was used. The Zivak Multitasker Fully Automated Sample Preparation and Injection System consist of a sample tray, vortex, centrifuge, column oven, UPLC pumps and an injection port. The system runs when the samples are placed in the sample tray together with the activation of the AUTOMASS software. $400 \mu \mathrm{L}$ of the calibrator, control and patient samples are transferred to the 2 $\mathrm{mL}$ vials by an automatic injector. After the addition of Reagent 1 and Reagent 2 , the mixture is vortexed for $1 \mathrm{~min}$ and centrifuged for 3 minutes at $5000 \mathrm{rpm}$. Then, $50 \mu \mathrm{L}$ of supernatant is injected to the column through a CSPE system. The results are calculated and sent to the data evaluation system within 2 minutes via AUTOMASS software. A more detailed description of the customized program of the ZIVAK Multitasker Vitamin D system is described in the manual for ZIVAK Vitamin D [15]. The sample chromatograms of the measured CRM (UME CRM 1308) $(51.00 \pm 2.92) \mathrm{ng} / \mathrm{mL}$ and $(49.76 \pm 2.65) \mathrm{ng} / \mathrm{mL}$ values of 25-OH-Vitamin D2 and D3, respectively, are shown in Figure 1.

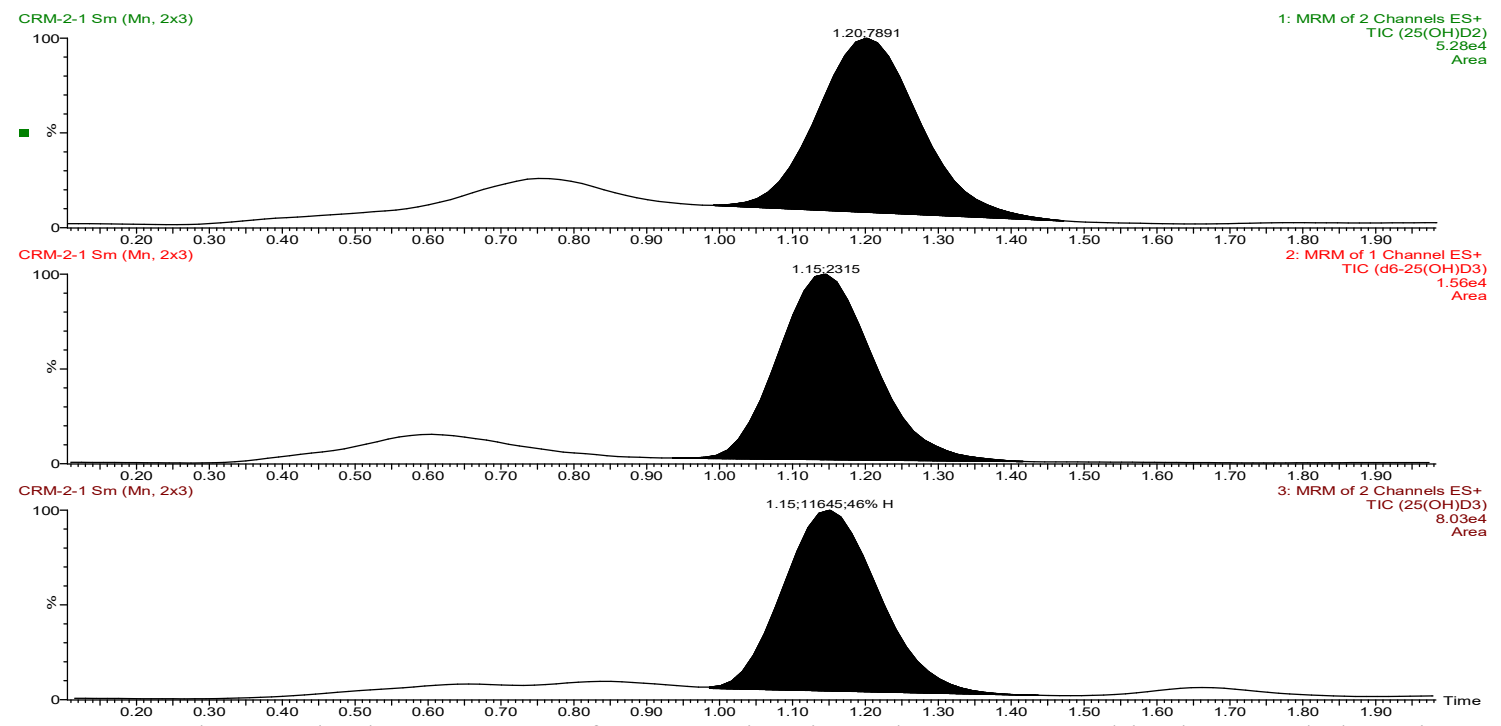

Figure 1. The sample chromatogram of 25-OH-Vitamin Ds by ZIVAK Multitasker coupled MS in UME

CRM 1308 


\section{Results and discussion}

\subsection{Method Validation}

The validation process was carried out based on the EURACHEM guideline $[4,13,16]$.

\subsubsection{Linearity and working range}

The linearity and working range of the method was evaluated by preparing the calibration solutions gravimetrically at four concentration levels and analyzing them in triplicate. Labelled standard was added into the solutions at constant concentration value. The calibration graph was plotted by placing the peak area ratios for native/labeled compounds on -y- axis and concentration of native standard on -x- axis. The linear regression and squared correlation coefficient was used to evaluate the linearity concentration levels and R2 values are shown in Table 3.

\subsection{2. $L O D$ and $L O Q$}

The evaluation of limit of detection (LOD) and limit of quantification (LOQ) was accomplished by analyzing the samples contains both D2 at $7 \mathrm{ng} / \mathrm{mL}$ and D3 at $10 \mathrm{ng} / \mathrm{mL}$ concentration levels. The LOD and LOQ values were obtained by multiplying the standard deviation (SD) of the results by 3 and 10 , respectively and were presented in Table 3 .

\subsubsection{Trueness}

The trueness was assessed by using UME CRM 1308. Method was applied in triplicate. The recovery values were calculated as a representative of trueness and presented in Table 3 . The obtained results of the measurement of UME CRM 1308 were presented in Table 4.

\subsubsection{Precision}

Precision consists of repeatability and intermediate precision sub parameters. They were assessed via standard addition method at three different concentration levels which are low, medium and high level of working range. Samples were prepared in triplicate. Repeatability was studied in a day and intermediate precision was studied in three days. The relative standard deviation values (RSD\%) were calculated to identify the precision and results were shown in Table 3.

Table 3. Method Validation Results

\begin{tabular}{lcccccccc}
\hline & \multicolumn{1}{c}{ Precision } \\
\hline & $\begin{array}{c}\text { Working } \\
\text { Range } \\
\left.(\mathrm{ngmL})^{-1}\right)\end{array}$ & $\begin{array}{c}\text { Linear Regression } \\
\text { Equation }\end{array}$ & $\mathrm{R}^{2}$ & $\begin{array}{c}\text { Recovery } \\
(\%)\end{array}$ & $\begin{array}{c}\text { Repeatability } \\
\text { RSD } \%\end{array}$ & $\begin{array}{c}\text { Intermediate } \\
\text { Precision } \\
\text { RSD \% }\end{array}$ & $\begin{array}{c}\text { LOD } \\
\left.(\mathrm{ngmL})^{-1}\right)\end{array}$ & $\begin{array}{c}\text { LOQ } \\
(\mathrm{ng} \\
\left.\mathrm{mL}^{-1}\right)\end{array}$ \\
25-OH-Vit. D2 & $9-45$ & $\mathrm{y}=0.074 \mathrm{x}+0.1645$ & 0.9966 & 86 & 5.66 & 7.85 & 2.62 & 8.73 \\
25-OH-Vit.D3 & $12-35$ & $\mathrm{y}=0.092 \mathrm{x}+0.2016$ & 0.9976 & 103 & 4.45 & 8.32 & 2.52 & 8.40 \\
\hline
\end{tabular}

Table 4. Measurement results and expanded uncertainty of UME CRM 1308 in ngmL $^{-1}$

\begin{tabular}{lcccc}
\hline & \multicolumn{2}{c}{ Certified Value \pm Uncertainty } & \multicolumn{2}{c}{ Measured Value \pm Uncertainty } \\
\hline Replicate Number & D3 & D2 & D3 & D2 \\
\hline CRM-R1 & $49.76 \pm 2.65$ & $51.00 \pm 2.92$ & $52.27 \pm 3.97$ & $43.31 \pm 3.34$ \\
CRM-R2 & $49.76 \pm 2.65$ & $51.00 \pm 2.92$ & $51.71 \pm 3.93$ & $42.95 \pm 3.31$ \\
CRM-R3 & $49.76 \pm 2.65$ & $51.00 \pm 2.92$ & $54.06 \pm 4.11$ & $42.24 \pm 3.25$ \\
\hline
\end{tabular}




\subsection{Measurement Uncertainty}

The bottom-up approach was applied to obtain measurement uncertainty value $[4,14,16]$. The sources for uncertainty were weighing of sample, calibration graph, trueness and precision.

\subsubsection{Weighing of the Sample}

Combined standard measurement uncertainty of sample was calculated using Equation 1 where $u_{c a l}$ was obtained from certificate of the balance supplied by the manufacturer.

$$
u(W)=\sqrt{\left(u_{\text {Calsample }}\right)^{2}+\left(u_{\text {Caltare }}\right)^{2}}
$$

where,

$u(W)$ : Combined standard measurement uncertainty of weighing sample $u_{\text {Calsample: }}$ Standard measurement uncertainty from calibration of balance while measuring sample $u_{\text {Caltare: }}$ Standard measurement uncertainty from calibration of balance while measuring tare

\subsubsection{Uncertainty of Calibration Graph}

The Equation 2 was used to calculate the combined standard measurement uncertainty of calibration graph.

$$
u\left(c_{0}\right)=\frac{S}{B_{1}} \sqrt{\frac{1}{p}+\frac{1}{n}+\frac{\left(c_{0}-\bar{c}\right)^{2}}{S_{x x}}} \quad S x x=\sum_{i=1}^{n}\left(c_{i}-\bar{c}\right)^{2}
$$

where,

$u\left(c_{0}\right)$ : Combined standard measurement uncertainty of calibration curve

$S:$ Residual standard deviation

B1: Slope

$p$ : Number of measurement to determine $\mathrm{c}_{0}$

$n$ : Number of measurement for calibration

$c_{0}$ : Determined concentration of the sample by using calibration curve

$c$ : Average value of the different calibration solution concentrations

\subsubsection{Uncertainty of Trueness}

The uncertainty value related to assessment of trueness was obtained based on the evaluation of recovery. UME-CRM 1309 produced by Reference Materials Laboratory of TUBITAK National Metrology Institute (UME) was used to evaluate the recovery of method regarding of vitamin D2 and D3. The method was applied on CRM in triplicates in a day and mean was calculated of the results. The uncertainty value of recovery was calculated by the Equation 3 .

$$
u\left(R_{m}\right)=R_{m} \sqrt{\left(\overline{\frac{u\left(C_{o b s}\right)}{\overline{C_{o b s}}}}\right)^{2}+\left(\frac{u\left(C_{c e r t}\right)}{C_{c e r t}}\right)^{2}}
$$




$$
R_{m}=\frac{\overline{C_{o b s}}}{C_{c e r t}}
$$

Where,

$u(R m)$ :Combined standard measurement uncertainty of recovery

$u\left(C_{o b s}\right)$ : Standard measurement uncertainty of observation of CRM or fortified material

$u\left(C_{c e r t}\right)$ : Standard measurement uncertainty of certification of CRM or fortified material

$R_{m}$ : Mean recovery

$C_{\text {obs: }}$ : Obtained concentration in recovery experiments

Ccert: Spiked or certified concentration

\subsubsection{Uncertainty of Precision}

The uncertainty originated from precision was evaluated via repeatability of the method. It was calculated by Equation 5.

$$
u(r)=\frac{R S D}{\sqrt{n}}
$$

where,

$u(r)$ : Standard measurement uncertainty of repeatability

$R S D$ : Relative standard deviation

$n$ : Number of sample

The Equation 6 is used for the calculation of combined standard measurement uncertainty of the vitamin D2 and D3 in serum. The expanded measurement uncertainty was obtained by multiplying the combined standard measurement uncertainty value with 2 (coverage factor) at $95 \%$ confidence level. The uncertainty budget of vitamin D2 and D3 are presented in Table 5 and Table 6.

$$
\frac{u_{c}(A)}{C_{A}}=\sqrt{\left(\frac{u\left(W_{S S}\right)}{W_{S S}}\right)^{2}+\left(\frac{u\left(c_{0}\right)}{c_{0}}\right)^{2}+u\left(R_{m}\right)^{2}+u(r)^{2}}
$$

where,

$u_{c}(A)$ : Combined standard measurement uncertainty of analyte

$C_{A}$ : Concentration of analyte

$u\left(W_{S S}\right)$ : Combined standard measurement uncertainty of sample intake

$W_{S S}$ : Weight of starting sample

$u\left(c_{0}\right)$ : Combined standard measurement uncertainty of calibration curve

$c_{0}$ : Determined concentration of the sample by using calibration curve

$u\left(R_{m}\right)$ : Combined standard measurement uncertainty of recovery

$u(r)$ : Standard measurement uncertainty of repeatability 
Table 5. Uncertainty budget of 25-OH-Vitamin D2 measurement method

\begin{tabular}{llccc}
\hline Parameters & Unit & Value $(\mathrm{X})$ & $\mathrm{u}(\mathrm{x})$ & $\mathrm{u}(\mathrm{x}) / \mathrm{X}$ \\
\hline Mass of sample intake & $\mathrm{g}$ & 10 & $2.1783 \mathrm{E}-05$ & $2.18 \mathrm{E}-06$ \\
Calibration & $\mathrm{ng} / \mathrm{mL}$ & 40.0 & 0.514 & $1.28 \mathrm{E}-02$ \\
Recovery & $\%$ & 0.840 & 0.027 & $3.26 \mathrm{E}-02$ \\
Repeatability & $\mathrm{Ng} / \mathrm{mL}$ & 42.83 & 0.6668 & $1.56 \mathrm{E}-02$ \\
& & & & \\
Relative Standard Measurement & & & & 0.038 \\
Uncertainty & & 42.8 & & \\
Result (ng/mL) & & & \\
Combined Standard Measurement & & & 3.3 & \\
Uncertainty & & & 7.7 & \\
Expanded Uncertainty (k=2) & & & \\
Relative Measurement Uncertainty (\%) & & & & \\
\hline
\end{tabular}

Table 6. Uncertainty budget of $25-\mathrm{OH}-\mathrm{Vitamin} \mathrm{D} 3$ measurement method

\begin{tabular}{llccc}
\hline Parameters & Unit & Value $(\mathrm{X})$ & $\mathrm{u}(\mathrm{x})$ & $\mathrm{u}(\mathrm{x}) / \mathrm{X}$ \\
\hline Mass of sample intake & $\mathrm{g}$ & 10 & $2.1783 \mathrm{E}-05$ & $2.18 \mathrm{E}-06$ \\
Calibration & $\mathrm{ng} / \mathrm{mL}$ & 40.0 & 0.405 & $1.01 \mathrm{E}-02$ \\
Recovery & $\%$ & 1.059 & 0.034 & $3.20 \mathrm{E}-02$ \\
Repeatability & $\mathrm{ng} / \mathrm{mL}$ & 52.68 & 0.9385 & $1.78 \mathrm{E}-02$ \\
& & & & \\
Relative Standard Measurement & & & & 0.038 \\
Uncertainty & & 52.7 & & \\
Result (ng/mL) & & & \\
Combined Standard Measurement & & & 4.0 & \\
Uncertainty & & & 7.6 & \\
Expanded Uncertainty (k=2) & & & \\
Relative Measurement Uncertainty (\%) & & & & \\
\hline
\end{tabular}

\section{Conclusions}

In this study it was shown that the ZIVAK Vitamin D analyzer can be successfully attached to a Waters Premier XE LC-MS/MS instrument and through the interface software control the MS. It was proven that the Vitamin D analyzer kit and sample preparation system can make highly accurate and reliable measurements with small uncertainties. Measurements of less than $<8 \%$ for $25-\mathrm{OH}$ vitamin $\mathrm{D} 2$ and $25-\mathrm{OH}$ vitamin D3 with widened uncertainty values were obtained (Table 4 and Table 5). The low LOD and LOQ values obtained with the ZIVAK Vitamin D analyzer are highly suitable and within the decision limit when considering measurements in clinical laboratory applications and their decision limits.

A recent study by NIST showed that, the consensus values of popular and commercially available techniques which are CLIA, EIA, RIA and LC are found to be higher than the NIST value which is defined by LC-ID/MS. Additionally, the applied method can detect 3-epi-25-OH-Vitamin D3 as well. The measurement results of the ZIVAK Vitamin D kit and Multitasker coupled MS agree with the LC-MS/MS measurements consensus value and the NIST expanded uncertainty range in the exercise.

This study clearly shows that, Zivak Multitasker Fully Automated Sample Preparation and Injection System connected to MS system gives the possibility of quick, accurate and reliable measurements of 25$\mathrm{OH}$ vitamin $\mathrm{D} 2$ and $25-\mathrm{OH}$ vitamin D3 with an LC-MS/MS in clinical laboratories for routine measurements. 


\section{Supporting Information}

Supporting Information accompanies this paper on http://www.acgpubs.org/JCM

\section{ORCID}

Ahmet C Goren: 0000-0002-5470-130X

Gözde Şahin: 0000-0001-9227-900X

İsmail Gümilcineli: 0000-0003- 2241-5904

Burcu Binici: 0000-0002-9616-8320

\section{References}

[1] M.F. Holick, in W.O.Song, G.R.Beecher, R.R.Eitenmiller and J.D. Winefordner (Eds.) (2000). Modern Analytical Methodoliges in Fat-and Water-Soluble Vitamins,( In Chemical Amalysis: A series of Monographs on Analytical Chemistry and Its Applications,Vol. 154), John Wiley \& Sons, Inc., New York, p.51.

[2] R. Belsey, M.B. Clark, M. Bernat, J. Glowacki, M.F. Holick, H.F. Deluca and J.T. potts (1974). physiologic significance of plasma transport of vitamin-d and metabolites, Am. J. Med., 57, 50-56.

[3] Z. Maunsell, D.J. Wright, S. J. Rainbow (2005). Routine isotope-dilution liquid chromatographytandem mass spectrometry assay for simultaneous measurement of the 25-hydroxy metabolites of vitamins D-2 and D-3, Clin. Chem., 1683-1690.

[4] a) A.C. Gören, G. Bilse and M. Bilsel (2007). Rapid and simultaneous determination of 25-OH-vitamin D2 and D3 in human serum by LC/MS/MS: Validation and uncertainty assessment, J.Chem.Metrol., 1, 1-9. b) H.O.Hamad, M. H. Alma, İ.Gulcin, M. A. Yılmaz and E. Karaoğul (2017). Evaluation of phenolic contents and bioactivity of root and nutgall extracts from Iraian Quercus infectoria Olivier, Rec.Nat.Prod. 11, 205210 .

[5] E.M. Mineva, R.L. Schleicher, M. Chaudhary-Webb, K.L. Maw, J.C. Botelho, H.W. Vesper and C.M. Pfeiffer (2015). A candidate reference measurement procedure for quantifying serum concentrations of 25hydroxyvitamin D3 and 25-hydroxyvitamin D2 using isotope-dilution liquid chromatography-tandem mass spectrometry, Anal. Bioanal. Chem., 407, 5615-5624

[6] K.W. Phinney, M. Bedner, S.C. Tai, V. Vamathevan, L.C. Sander,K.E. Sharpless, S.A. Wise, J.H. Yen, R.L. Schleicher, M. Chaudhary-Webb, J.M. Betz, P.M.Coates and M.F. Picciano, (2002) Development and Certification of a Standard Reference Material for Vitamin D Metabolites in Human Serum, Anal. Chem., 84, 956-962.

[7] M. Bedner and K. A. Lippa (2012). NIST/NIH Vitamin D Metabolites Quality Assurance Program Report of Participant Results Winter 2012 Exercise. P.1-23.

[8] M. Bedner and K. A. Lippa (2013). NIST/NIH Vitamin D Metabolites Quality Assurance Program Report of Participant Results Summer 2013Comparability Study p.1-23.

[9] S. A. Wise, S.C. Tai, D.L. Duewer, M. Bedner, J.E. Camara, K. A Lippa, L. Qinde, D. Kang, B. Kim, C. Quan, L.Shi, J. Nammoonnoy, V. Vamathevan, A. C. Gören, G. Bilsel and H. Yilmaz (2017). CCQM-K132: low-polarity analytes in a biological matrix: vitamin D metabolites in human serum, Metrologia, 54S, https://doi.org/10.1088/0026-1394/54/1A/08027

[10] Certificate of Analysis, SRM 972 Vitamin D in Human Serum, National Institute of Standards and Technology (NIST), June 9, 2009. https://www-s.nist.gov/srmors/view cert.cfm?srm=972

[11] Certificate of Analysis, SRM 972a Vitamin D Metabolites in Frozen Human Serum, National Institute of $\begin{array}{llllll}\text { Standards and Technology } & \text { (NIST), } & \text { February } & \text { 2013. }\end{array}$ s.nist.gov/srmors/view cert.cfm?srm=972A

[12] Certification Report, UME CRM 1308, 25-Hydroxy Vitamin D2 and 25-Hydroxy Vitamin D3 in Lyophilized Serum, UME TÜBİTAK, Gebze-Kocaeli, Turkey 9. Certificate of Analysis, SRM 2973 Vitamin D Metabolites in Frozen Human Serum (High Level), National Institute of Standards and Technology (NIST), February 4, 2016. https://www-s.nist.gov/srmors/view cert.cfm?srm=2973

[13] EURACHEM, The Fitness for Purpose of Analytical Methods A Laboratory Guide to Method Validation and Related Topics, 2014.

[14] EURACHEM/CITAC Guide CG 4 Editors : S L R Ellison, A Williams. Quantifying Uncertainty in Analytical Measurement, 3rd ed., 2012. 
[15] ZIVAK Technologies, 25-Hydroxyvitamin D2-D3 Serum LC-MS/MS APCI Analysis Kit User Manual, D. No: ZV-3046-KK-10.R12.

[16] B. Binici, M. Bilsel, M. Karakas, I. Koyuncu, A.C. Goren (2013) An efficient GC-IDMS method for determination of PBDEs and PBB in plastic materials, Talanta, 116,417-426.

$$
\text { A } \underset{\text { publications }}{\mathbf{C}}
$$

(C) 2018 ACG Publications 